\title{
Metformin combined with acarbose vs. single medicine in the treatment of type 2 diabetes: A meta-analysis
}

\author{
ZHONGJIE LIU ${ }^{1}$, XIAODONG ZHAO ${ }^{2}$, WEIWEI SUN ${ }^{1}$, YAOXIAN WANG ${ }^{1}$, SHANGJIAN LIU ${ }^{1}$ and LEI KANG $^{2}$ \\ Departments of ${ }^{1}$ Nephropathy and Endocrinology, and ${ }^{2}$ Encephalopathy, \\ Dongzhimen Hospital Affiliated to Beijing University of Chinese Medicine, Beijing 100700, P.R. China
}

Received August 4, 2015; Accepted January 6, 2017

DOI: $10.3892 /$ etm.2017.4333

\begin{abstract}
The present meta-analysis aimed to evaluate metformin combined with acarbose compared with monotherapy with either of the two drugs for type 2 diabetes (T2DM). Relevant trials were retrieved through searching PubMed, Embase, Cochrane library, China National Knowledge Infrastructure, Wanfang and Chongqing VIP information network databases. Heterogeneous and homogeneous data were statistically combined using a random- and fixed-effects model, respectively. For dichotomous and continuous data, the merged effect size was presented as the risk ratio (RR) and weighted mean differences (WMD), respectively, with $95 \%$ confidence interval (CI). All included studies were divided into subgroups. A Funnel plot was used to detect publication bias. Review Manager 5.2 software was applied to perform the statistical analyses. Meta-analysis revealed that compared with metformin monotherapy, combined therapy was significantly more efficacious regarding indexes including the total effective rate, fasting blood glucose (FBG), blood glucose levels at two post-prandial hours (2HPG) and hemoglobin A1C (HbAlc). Similarly, combined therapy showed advantages on indexes including FBG, 2HPG and HbA1c over acarbose therapy after 4 months of treatment. In conclusion, the findings of the present meta-analysis suggested that combined therapy of metformin and acarbose appears to be more efficacious than metformin or acarbose monotherapy.
\end{abstract}

\section{Introduction}

Type 2 diabetes (T2DM), also known as non-insulin-dependent diabetes mellitus, is a chronic metabolic disorder

Correspondence to: Dr Xiaodong Zhao, Department of Encephalopathy, Dongzhimen Hospital Affiliated to Beijing University of Chinese Medicine, 5 Haiyuncang Hutong, Dongcheng, Beijing 100700, P.R. China

E-mail: xzh_dong@163.com

Key words: acarbose, metformin, type 2 diabetes, meta-analysis caused by the body's inadequate production or use of insulin and results in excessive amounts of glucose in the blood and urine $(1,2)$. T2DM may have various complications affecting the nervous system, the eye and the kidneys (3-5). Various health indicators, including total effective rate $(\%=$ excellence+improvement $] /$ total cases x 100), fasting blood glucose (FBG) and blood glucose levels at two post-prandial hours (2HPG) give information on the efficacy of medications for T2DM. Not all studies indicated a total effective rate. In addition, blood lipid indexes, including hemoglobin A1C (HbA1c), triglyceride (TG), total cholesterol (TC), high-density lipoprotein (HDL) and low-density lipoprotein (LDL) may be used as health indicators. Various medicines have been assessed for the treatment of T2DM, including sulfonylureas, glinides, biguanides and $\alpha$-glycosidase inhibitor (6-9); however, no final conclusion has yet been reached on the best treatment of T2DM.

Acarbose and metformin treatment has been reported to have a beneficial effect on patients with T2DM $(10,11)$. Metformin, which is the most frequently prescribed oral hypoglycemic agent, targets insulin resistance and excessive glucose $(12,13)$. However, $\beta$-cell function continues to deteriorate in patients with $\mathrm{T} 2 \mathrm{DM}$, accompanied with progressive failure of insulin secretion (14). The development of T2DM has been shown to be delayed by acarbose by impairing glucose intolerance (15). However, acarbose has common gastrointestinal adverse effects, including abdominal pain, diarrhea and bloating (16). With the increasing promotion of medicine combinations, various studies have provided gradually increasing evidence that acarbose combined with metformin treatment has a higher efficacy than monotherapy (17-19). It has also been indicated that combined medicines have higher efficacy with regard to HbA1c, FBG and $2 \mathrm{HPG}$ as well as insulin levels (19). At the same time, this therapy method is safe and well tolerated. However, the results of these studies were not statistically significant. Particularly in China, the inconsistencies of results are more obvious due to various factors, including heredity, immune system, environment, region and ethnicity. In addition, the low number of samples assessed by individual clinical trials has represented a limitation.

Therefore, the aim of the present meta-analysis was to assess and compare the advantageous effects of acarbose combined with metformin treatment over monotherapy using either drug in the treatment of T2DM. By retrieving relevant 
studies using Chinese Han populations and performing a meta-analysis, evidence-based results on a large number of samples were provided.

\section{Materials and methods}

Literature search. The electronic databases Pubmed (http://www.ncbi.nlm.nih.gov/pubmed/), Embase (http://www.elsevier.com/online-tools/embase), Cochrane library (http://www.cochranelibrary.com/), China National Knowledge Infrastructure database (CNKI; http://www.cnki. net/), Wanfang database (http://www.wanfangdata.com.cn/) and Chongqing VIP information network (http://www.cqvip. $\mathrm{com} /$ ) were searched for studies on metformin combined with acarbose or the respective monotherapies for the treatment of (T2DM) published until March 2015. The following keywords were used for search strategy: 'Acarbose', 'metformin', 'type 2 diabetes' and the Chinese equivalent.

Inclusion and exclusion criteria. Studies included in the present meta-analysis were selected using the following criteria: i) Chinese Han populations with T2DM as the subjects; ii) randomized clinical trials designed to compare the effects of combined metformin and acarbose vs. monotherapy for T2DM; iii) T2DM diagnosed according to the standards of the World Health Organization (WHO) (20); iv) inclusion of at least one of the following evaluation indexes: Total effective rate, FBS, 2HPG and HbA1c; v) sufficient information for calculation of merged effect size; and vi) among various studies with duplicate data, the study with the higher quality and number of indexes assessed was selected.

Studies were excluded for the following reasons: i) No specific description of treatment course included in the study; ii) literature reviews, meeting reports and letters.

Data extraction and quality assessment. Two evaluators assessed the quality of all studies and extracted relevant data independently. For each article retrieved, the following data were extracted: Name of first author, year of publication, region where the study was performed, the number of patients, age of the experimental and control groups, treatment course of diabetes, dosage regimen, evaluation index (total effective rate, FBG, 2HPG, HbAlc; the mean \pm standard deviation was calculated). Any disagreement was resolved by discussion with a third investigator. The Cochrane quality evaluation system was used to assess the quality of the included studies (21).

Statistical analysis. Review Manger 5.2 software (Cochrane Collaboration, Oxford, UK) was used for meta-analysis in this study. $\chi^{2}$-based Q test (22) and $I^{2}$ statistics were chosen for heterogeneity test. $\alpha=0.05$ was set as a testing standard. The fixed-effects model (Mantel-Haenszel method) was used for meta-analysis when the heterogeneity inspection results were $\mathrm{P}>0.05$ and $I^{2}<50 \%$. Otherwise, the random-effects model (Dersimonian-Laird method) was used. For dichotomous data, the merged effect size was presented as the risk ratio (RR) with $95 \%$ confidence interval (CI). For continuous data, the merged effect size was shown as weighted mean differences (WMD) with 95\% CI. All included studies were divided into subgroups for further analysis based on different treatment

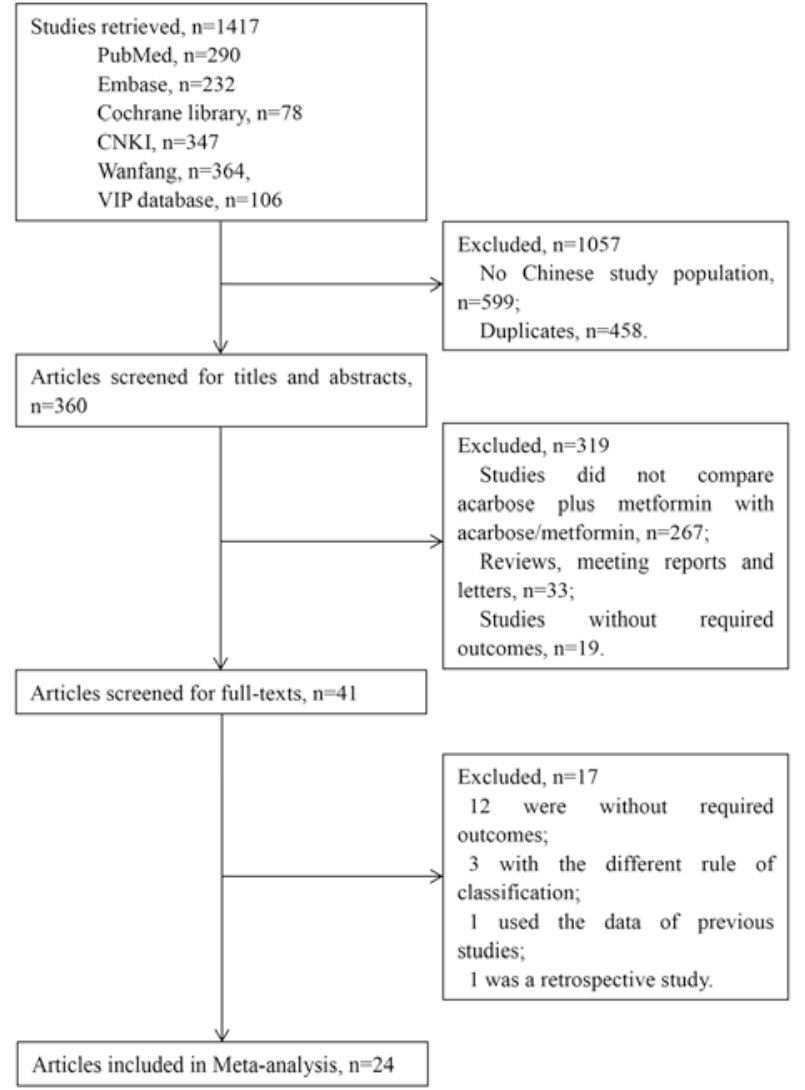

Figure 1. Flow chart of literature search and study selection. CNKI, China National Knowledge Infrastructure; VIP, Chongqing VIP information network.

courses. Funnel plots were used to detect publication bias. Sensitivity analysis was applied to test the stability of results by eliminating low-quality studies and combing other studies.

\section{Results}

Characteristics of studies included. Initially, a total of 1,417 studies were retrieved (290 with pubmed, 232 with embase, 78 with the Cochrane, 347 with the NCKI, 364 with the Wanfang and 106 with the VIP database) based on the key words. Among them, 1,057 studies were duplicates or not focused on Chinese Han populations. After screening of the abstracts, the full-text versions of 41 studies were then retrieved. Among these, a total of 17 studies were excluded, including 12 studies lacking the required data, 3 studies with the different rule of classification, 1 study with duplicate data and 1 retrospective study. Finally, a total of 24 relevant studies were selected for meta-analysis (23-46) (Fig. 1). The included studies contained 1 English language study and 23 Chinese language studies comprising 2,337 patients. As shown in Table I, these patients were treated for durations of 2 weeks, 2 , 3, 4 and 6 months. And the treatment duration of the majority of patients was 3 months. For most patients, the regimen for acarbose was $50 \mathrm{mg}$ three times a day during meals, while that for metformin was $500 \mathrm{mg}$ three times a day after meals. After the studies included were assessed by the Cochrane quality evaluation system, the study by Wang et al (36) was confirmed to be a high-quality study with a low risk of bias. 


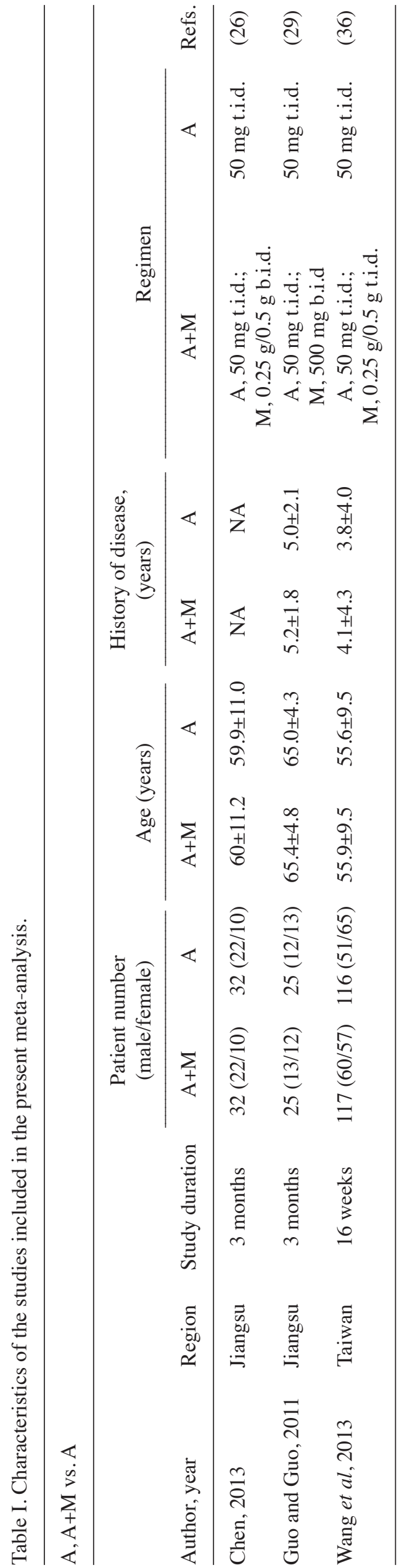











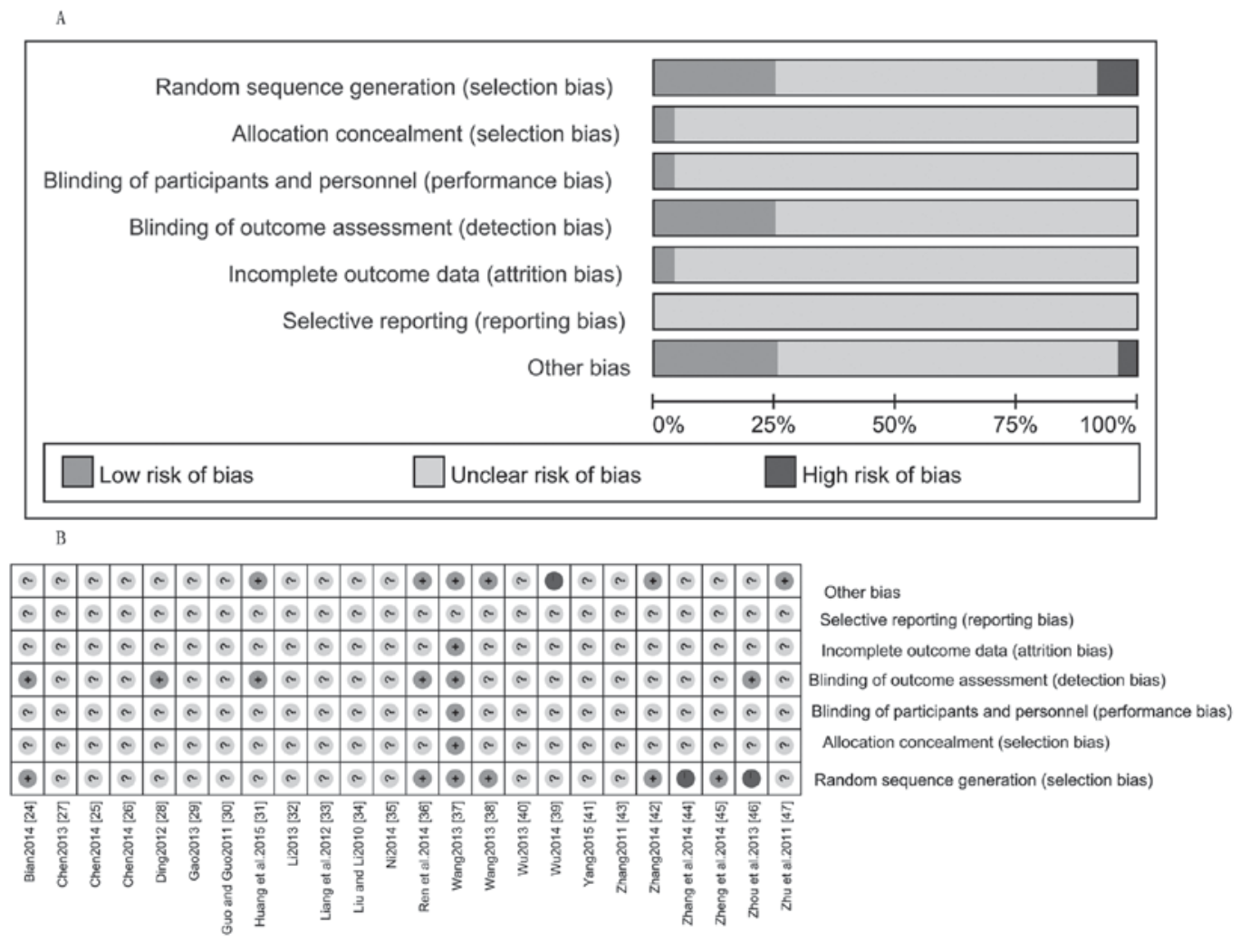

Figure 2. (A) Risk of bias graph and (B) bias summary.

However, unclear risk of bias was determined for the other studies (Fig. 2A and B).

Meta-analysis of combined therapy compared with metformin monotherapy for T2DM. A total of 21 studies including 1,968 patients (983 receiving combined therapy and 985 receiving monotherapy) reported on combined therapy vs. metformin monotherapy for T2DM patients $(23-25,27,28$, 30-35,37-46).

Total effective rate. The heterogeneity test showed no significant differences between studies regarding the total effective rate $\left(\mathrm{P}>0.05, I^{2}<50 \%\right)$. Therefore, the fixed-effects model was applied to calculate the merged effect size. Regarding the total effective rates for combined therapy vs. metformin monotherapy for T2DM patients for 6 months, 3 months, 2 months and 2 weeks, the RRs were 1.19 (95\% CI: 1.07, 1.33; P=0.002), 1.22 (95\% CI: 1.14, 1.30; P<0.01), 1.26 (95\% CI: 1.14, 1.38; $\mathrm{P}<0.01)$ and 1.44 (95\% CI: 1.20, 1.73; $\mathrm{P}<0.01)$, respectively. As all differences were statistically significant, the combined therapy was shown to be a more effective treatment for T2DM than metformin monotherapy. In addition, the total effective rate was not significantly affected by the treatment duration as there was no significant difference between the total effective rate among different treatment durations ( $\mathrm{P}>0.05$; Fig. $3 \mathrm{~A})$.

Blood glucose. The indexes associated with blood glucose included FBG, 2HPG and HbA1c. The heterogeneity test indicated significant differences in FBG levels between studies $\left(\mathrm{P}<0.05, I^{2}>50 \%\right.$; Fig. 3B). Therefore, the random-effects model was applied to calculate the merged effect size. The
WMDs for combined therapy vs. metformin monotherapy for T2DM patients treated for 6 months, 3 months, 2 months and 2 weeks were -0.47 (95\% CI: 1.43, 0.49; P=0.34), -1.46 (95\% CI: $-2.41,-0.52 ; \mathrm{P}<0.01),-0.97$ (95\% CI: $-1.38,-0.56 ; \mathrm{P}<0.01)$ and -2.97 (95\% CI: $-3.86,-2.08 ; \mathrm{P}<0.01)$, respectively. The results showed that except for the 6-months group, the differences at were statistically significant for all other treatment durations.

Heterogeneity tests revealed significant differences in $2 \mathrm{HPG}$ among the studies ( $\mathrm{P}<0.05, I^{2}>50 \%$; Fig. $\left.3 \mathrm{C}\right)$. Therefore, the random-effects model was applied to calculate the merged effect size. The WMDs for combined therapy vs. metformin monotherapy for T2DM patients treated for 6 months, 3 months, 2 months and 2 weeks were -1.71 (95\% CI: -3.39 , $-0.02 ; \mathrm{P}=0.05),-1.62$ (95\% CI: $-2.91,-0.33 ; \mathrm{P}=0.01),-1.12$ (95\% CI: $-2.06,-0.18 ; \mathrm{P}=0.02)$ and -2.37 (95\% CI: $-3.38,-1.36$; $\mathrm{P}<0.01)$, respectively. The differences were not statistically significant with the exception of the 2-week group.

Heterogeneity tests revealed significant differences in HbAlc among the studies $\left(\mathrm{P}<0.05, I^{2}>50 \%\right.$; Fig. 3D). Therefore, the random-effects model was applied to calculate the merged effect size. The WMDs for combined therapy vs. metformin monotherapy for T2DM patients treated for 6, 3 and 2 months was -0.13 (95\% CI: $-0.61,0.35 ; \mathrm{P}=0.65),-0.80$ (95\% CI: -2.16 , $0.56 ; \mathrm{P}=0.25)$ and $-0.63(95 \% \mathrm{CI}:-1.07,-0.18 ; \mathrm{P}=0.006)$, respectively. The differences were statistically significant after two months, but not after three or six months of treatment.

Meta-analysis of combined therapy compared with acarbose monotherapy for T2DM. A total of 5 studies including 470 patients $(235$ receiving combined therapy and 235 receiving monotherapy) reported on the combined therapy vs. acarbose 


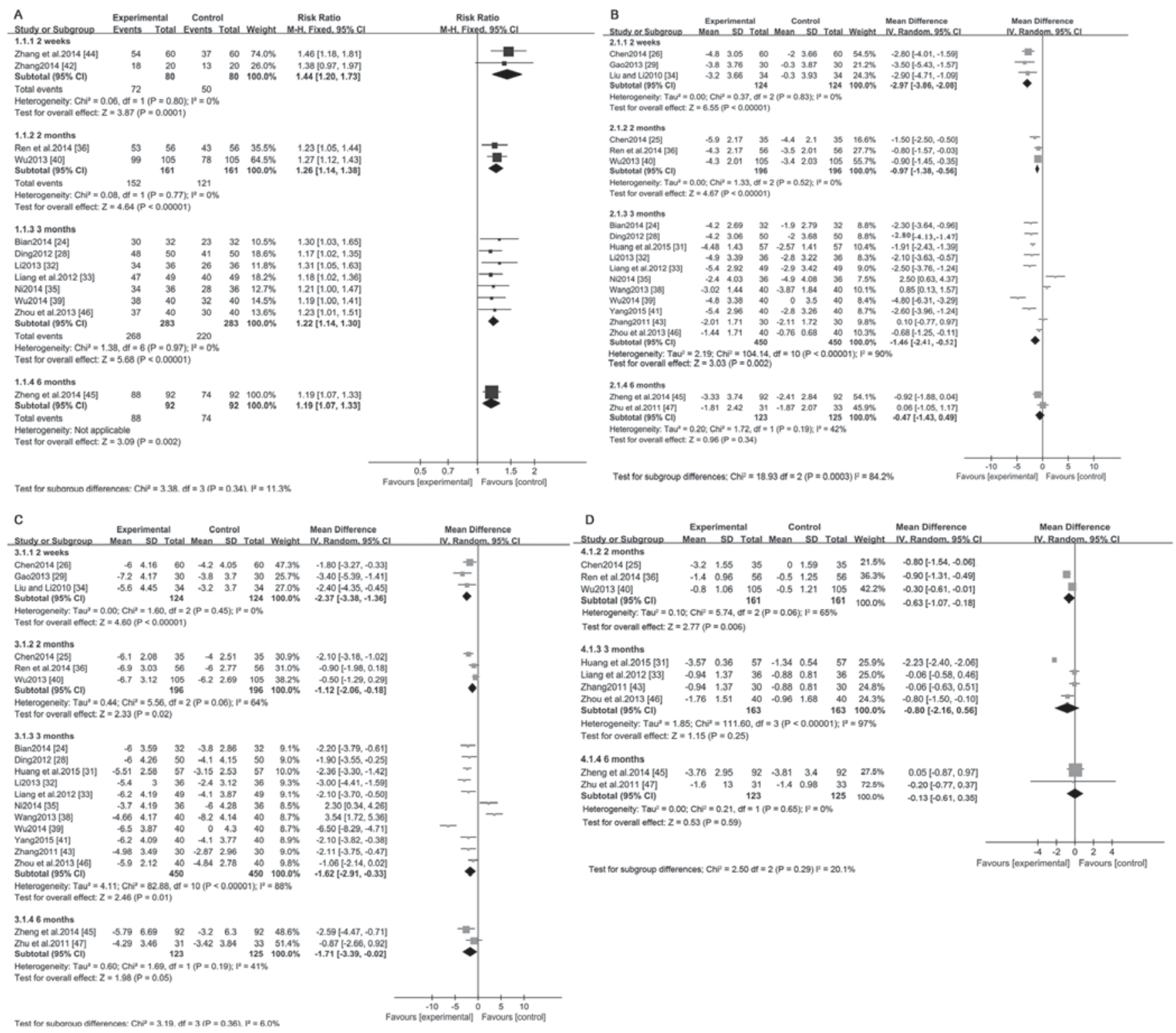

Figure 3. Forest plots of various indexes compared between combined therapy and metformin monotherapy. (A) Total effective rate; (B) fasting blood glucose; (C) blood glucose levels at two post-prandial hours; and (D) hemoglobin A1C. CI, confidence interval; df, degrees of freedom; M-H, Mantel-Haenszel; SD, standard deviation.

monotherapy for T2DM patients $(26,29,36,42,46)$. Based on the treatment course, only one study was available for each subgroup. Therefore, no meta-analysis was performed here for the included studies (Fig. 4A-D). The RR regarding the total effective rate of combined therapy vs. acarbose monotherapy for T2DM patients treated for 3 months was 1.29 (95\% CI: $1.05,1.59)$ and there was no statistically significant difference between the two groups. However, FBG, 2HPG and HbA1C at four months of treatment were significantly different between the two groups with WMDs of -1.61 (95\% CI: $-2.23,-0.99)$, -2.08 (95\% CI: $-3.21,-0.95)$ and -1.36 (95\% CI: $-1.66,-1.06)$, respectively.

Evaluation of publication bias. In the present study, funnel plots were used to identify publication bias. Symmetric funnel plots on the total effective rate and FBG showed that there was no publication bias in the studies included (Fig. 5A and B).
Sensitivity analysis. By eliminating low-quality studies $(38,43,45)$ in turn and combing other studies, it was confirmed that the results of the sensitivity analysis were reliable and stable.

\section{Discussion}

The present meta-analysis showed for the first time, to the best of our knowledge, that metformin combined with acarbose treatment of T2DM patients of a Chinese Han population had a greater beneficial effect than monotherapy with either drug. Acarbose is the first-line medication for the treatment of T2DM, which delays carbohydrate absorption by inhibiting $\alpha$-glycosidase enzymes on the surface of intestinal epithelial cells to effectively lower post-prandial hyperglycemia (47). Similarly, metformin enhances insulin sensitivity and decreases insulin resistance by increasing glucose uptake and utilization of peripheral tissue, accelerating anaerobic 


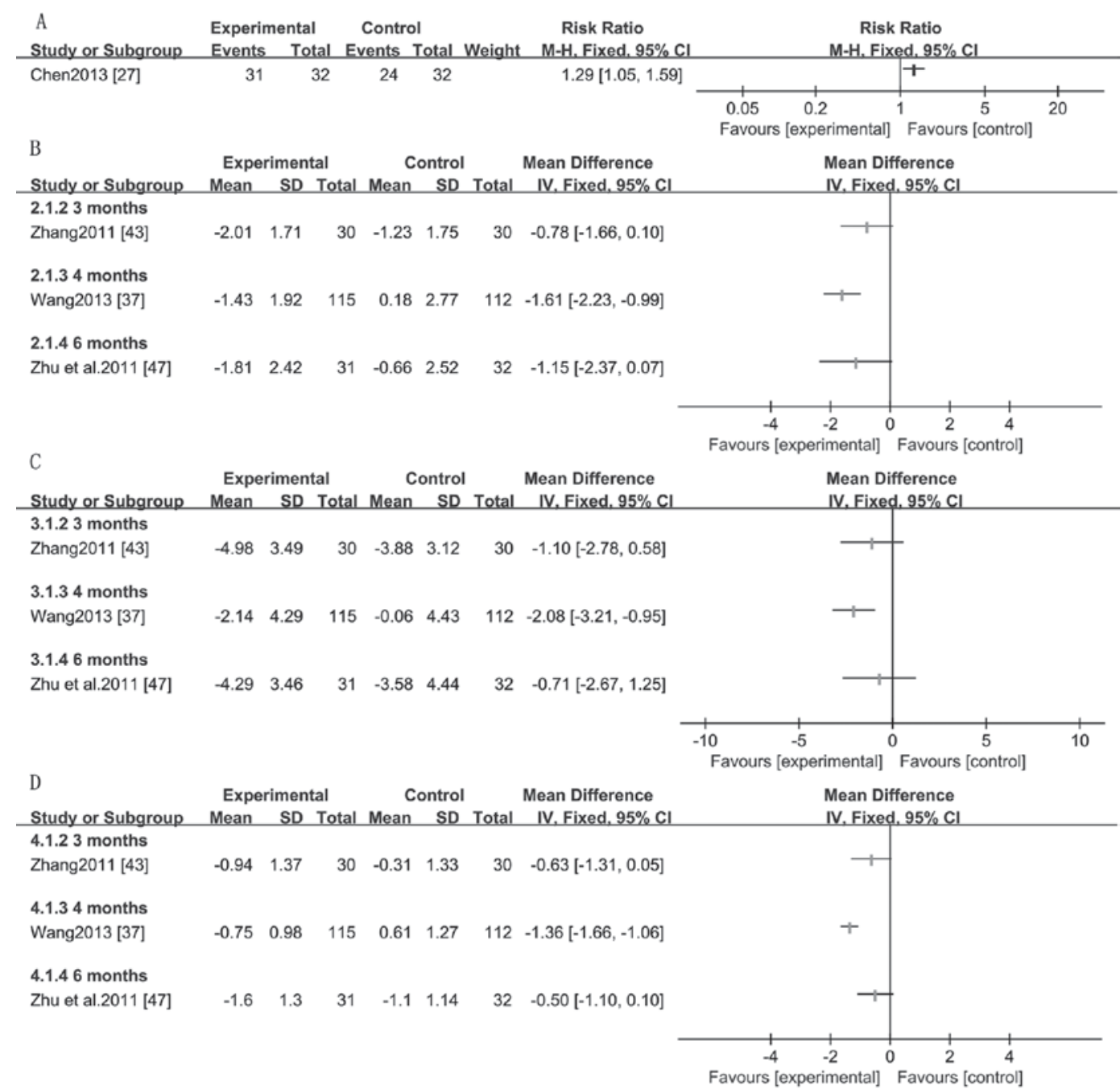

Figure 4. Forest plots of various indexes compared between combined therapy and acarbose monotherapy. (A) Total effective rate; (B) fasting blood glucose; (C) blood glucose levels at two post-prandial hours; and (D) hemoglobin A1C. CI, confidence interval; SD, standard deviation.
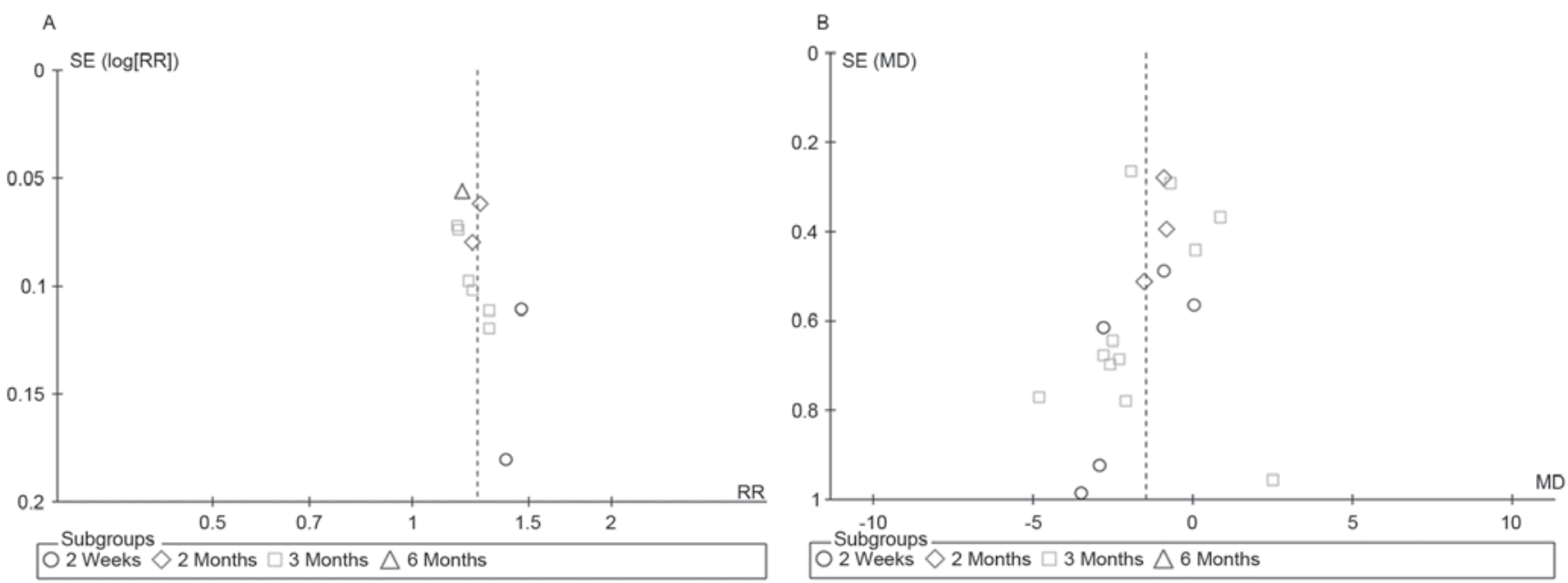

Figure 5. Funnel plot for (A) total effective rate and (B) fasting blood glucose. SE, standard error; RR, risk ratio; MD, mean difference.

glycolysis to inhibit glycogenosis, and reduce the output of hepatic glycogen (48). Therefore, metformin is considered to have various effects in metabolic regulation through activating the protein kinase signaling system (49). The actions of metformin and acarbose are complementary, and combined therapy may therefore achieve improved effects. Through reducing gluconeogenesis, metformin lowers the rate of glucose production (50). However, the results of the present 
study showed that the efficacy of this treatment method was lower than that of combined treatment after three months.

In the present meta-analysis, differences in indexes, including the total effective rate, fasting blood glucose, $2 \mathrm{HPG}$ and HbA1c were found between combined and monotherapy groups. The results were in accordance with the finding of most individual studies. In the study by Zhang (42), the combined treatment confirmed to be effective than single medicine treatment on various indexes such as FPG, 2HPG and BMR. A variety of previous meta-analyses on different therapy methods for T2DM patients are available. Boulé et al (51) confirmed that exercise therapy is an important constituent of diabetes treatment, which was able to reduce HbAlc as well as the risk of diabetic complications. Their meta-analysis study showed that physical therapy was beneficial for T2DM patients, while the efficacy of anti-diabetic drugs, particularly combined regimens, was demonstrated in the present study. Furthermore, a meta-analysis study by Amori et al (52) showed that incretin can be applied for T2DM with a favorable weight-change profile and modest efficacy. However, the present study did not perform any meta-analyses for indexes including lipid profile, postprandial glycemia and antibody development due to insufficient data provided by the studies included. Due to the selection criteria applied after the literature search, the studies included in the present meta-analysis contained comprehensive data on indexes including total effective rate, fasting blood-glucose, $2 \mathrm{HPG}$ and HbAlc.

Of note, the present meta-analysis had certain limitations. After the studies included were assessed using the Cochrane quality evaluation system, the study by Wang et al (36) was confirmed to be a high-quality study with low risk of bias. However, other studies $(38,43,45)$ had an unclear risk of bias and are therefore a lower standard. Furthermore, Fig. 5 indicated that there may have been bias for each individual time-point. In addition, only one study on combined vs. acarbose monotherapy was available for each subgroup based on the treatment course; therefore, the studies on combined therapy vs. acarbose monotherapy were not subjected to meta-analysis.

The present meta-analysis indicated that combined treatment with metformin and acarbose is more efficacious in T2DM patients than monotherapy with metformin or acarbose. In addition, with increasing treatment time (2 weeks, 2, 3 and 6 months), the differences in the efficacy between combined therapy and metformin monotherapy decreased. For clinical application, specific conditions of individual patients should be taken into account. In order to improve the therapeutic efficacy, combined therapy of metformin and acarbose is a better treatment method than monotherapy if it is matched to the individual patient.

\section{References}

1. Knowler WC, Barrett-Connor E, Fowler SE, Hamman RF, Lachin JM, Walker EA and Nathan DM; Diabetes Prevention Program Research Group: Reduction in the incidence of type 2 diabetes with lifestyle intervention or metformin. N Engl J Med 346: 393-403, 2002

2. DeFronzo RA: Pharmacologic therapy for type 2 diabetes mellitus. Ann Intern Med 131: 281-303, 1999.
3. National Collaborating Centre for Chronic Conditions: Type 2 Diabetes: National Clinical Guideline for Management in Primary and Secondary Care (Update). Royal College of Physicians, London, 2008.

4. Intensive blood-glucose control with sulphonylureas or insulin compared with conventional treatment and risk of complications in patients with type 2 diabetes (UKPDS 33). UK Prospective Diabetes Study (UKPDS) Group. Lancet 352: 837-853, 1998.

5. Nathan DM, Buse JB, Davidson MB, Ferrannini E, Holman RR, Sherwin R and Zinman B; American Diabetes Association; European Association for Study of Diabetes: Medical management of hyperglycemia in type 2 diabetes: A consensus algorithm for the initiation and adjustment of therapy: A consensus statement of the American diabetes association and the European association for the study of diabetes. Diabetes Care 32: 193-203, 2009.

6. Arai K, Matoba K, Hirao K, Matsuba I, Takai M, Takeda H, Kanamori A, Yamauchi M, Mori H and Terauchi Y: Present status of sulfonylurea treatment for type 2 diabetes in Japan: Second report of a cross-sectional survey of 15,652 patients. Endocr J 57: 499-507, 2010.

7. Füchtenbusch M, Standl E and Schatz H: Clinical efficacy of new thiazolidinediones and glinides in the treatment of type 2 diabetes mellitus. Exp Clin Endocrinol Diabetes 108: 151-163, 2000.

8. Bosi E: Metformin-the gold standard in type 2 diabetes: What does the evidence tell us? Diabetes Obes Metab 11 (Suppl 2): S3-S8, 2009.

9. Mitrakou A, Tountas N, Raptis AE, Bauer RJ, Schulz H and Raptis SA: Long-term effectiveness of a new alpha-glucosidase inhibitor (BAY m1099-miglitol) in insulin-treated type 2 diabetes mellitus. Diabet Med 15: 657-660, 1998.

10. Wang JS, Lin SD, Lee WJ, Su SL, Lee IT, Tu ST, Tseng YH, Lin SY and Sheu WH: Effects of acarbose versus glibenclamide on glycemic excursion and oxidative stress in type 2 diabetic patients inadequately controlled by metformin: A 24-week, randomized, open-label, parallel-group comparison. Clin Ther 33: 1932-1942, 2011.

11. Willms B and Ruge D: Comparison of acarbose and metformin in patients with type 2 diabetes mellitus insufficiently controlled with diet and sulphonylureas: A randomized, placebo-controlled study. Diabet Med 16: 755-761, 1999.

12. Setter SM, Iltz JL, Thams J and Campbell RK: Metformin hydrochloride in the treatment of type 2 diabetes mellitus: A clinical review with a focus on dual therapy. Clin Ther 25: 2991-3026, 2003.

13. Hundal RS and Inzucchi SE: Metformin: New understandings, new uses. Drugs 63: 1879-1894, 2003.

14. Nauck MA, Meininger G, Sheng D, Terranella L and Stein P; Sitagliptin Study 024 Group: Efficacy and safety of the dipeptidyl peptidase-4 inhibitor, sitagliptin, compared with the sulfonylurea, glipizide, in patients with type 2 diabetes inadequately controlled on metformin alone: A randomized, double-blind, non-inferiority trial. Diabetes Obes Metab 9: 194-205, 2007.

15. Chiasson JL, Josse RG, Gomis R, Hanefeld M, Karasik A and Laakso M; STOP-NIDDM Trail Research Group: Acarbose for prevention of type 2 diabetes mellitus: The STOP-NIDDM randomised trial. Lancet 359: 2072-2077, 2002.

16. Yee HS and Fong NT: A review of the safety and efficacy of acarbose in diabetes mellitus. Pharmacotherapy 16: 792-805, 1996.

17. Halimi S, Le Berre M and Grangé V: Efficacy and safety of acarbose add-on therapy in the treatment of overweight patients with type 2 diabetes inadequately controlled with metformin: A double-blind, placebo-controlled study. Diabetes Res Clin Pract 50: 49-56, 2000.

18. Phillips P, Karrasch J, Scott R, Wilson D and Moses R: Acarbose improves glycemic control in overweight type 2 diabetic patients insufficiently treated with metformin. Diabetes Care 26: 269-273, 2003.

19. Rosenstock J, Brown A, Fischer J, Jain A, Littlejohn T, Nadeau D, Sussman A, Taylor T, Krol A and Magner J: Efficacy and safety of acarbose in metformin-treated patients with type 2 diabetes. Diabetes Care 21: 2050-2055, 1998.

20. Grimaldi A and Heurtier A: Diagnostic criteria for type 2 diabetes. Rev Prat 49: 16-21, 1999 (In French).

21. Higgins JP and Green S (eds): Cochrane Handbook for Systematic Reviews of Interventions. Version 5.0.0 [updated February 2008]. The Cochrane Collaboration, 2008.

22. Lau J, Ioannidis JP and Schmid CH: Quantitative synthesis in systematic reviews. Ann Intern Med 127: 820-826, 1997.

23. Bian YH: Efficacy of acarbose and metformin in treatment of type 2 diabetes. China Modern Doctor 119-123,2014 (In Chinese). 
24. Chen HY: Effect observation of metformin combined with acarbose in the treatment of type 2 diabetes mellitus. Modern Medicine J China 21: 80-81, 2014 (In Chinese).

25. Chen LF: Efficacy and safety evaluation of acarbose combined with metformin in the treatment of type 2 diabetes. Clin Med Engineering 21: 1563-1564, 2014 (In Chinese).

26. Chen Y: Efficacy of joint of acarbose and metformin on the treatment of type 2 diabetes mellitus with hyperlipidemia. Chin J Mod Drug 7: 182, 2013 (In Chinese).

27. Ding XY: Analysis on efficacy and safety of acarbose combined metformin in treatment of type 2 diabetes. China Pharmaceuticals 21: 97-98, 2012 (In Chinese).

28. Gao Z: Clinical observation of metformin in combination with acarbose in the treatment of type 2 diabetes. Chinese J Urban Rural Enterprises Health 12: 45-46, 2013 (In Chinese).

29. Guo XH and Guo XZ: Efficacy of combination of metformin and acarbose on the treatment of type 2 diabetes mellitus with hyperlipidemia. China Health Industry 8: 54-55, 2011 (In Chinese).

30. Huang L, Liao B, Yang M, Liu JH, Quan HM and Qin AP: Acarbose in combination with metformin for treatment of incipient type 2 diabetes mellitus with hyperlipemia. Med Aesthetic Beauty 1: 211, 2015 (In Chinese).

31. Li L: The clinical efficacy of acarbose with metformin in the treatment of type 2 diabetes. Chinese Foreign Med Res 11: 13-14, 2013 (In Chinese)

32. Liang JH, Chen LZ and Gong ZZ: Efficacy of metformin extended release tablets combined with acarbose on the treatment of obesity in type 2 diabetes. J Guangd Med College 30: 192-193, 2012 (In Chinese)

33. Liu LL and Li GR: Efficacy of metformin with acarbose in the treatment of type 2 diabetes. Qingdao Med J 42: 265-266, 2010 (In Chinese).

34. Ni HH: Treatment effect of acarbose in combination with metformin for patients with type 2 diabetes. Strait Pharmaceutical J 11: 194-195, 2014 (In Chinese).

35. Ren Y, Jiao F and Bai Y: Clinical curative effect of metformin in combination with acarbose for the treatment of type 2 diabetes. China Health News Med 19: 26-26, 2014 (In Chinese).

36. Wang JS, Huang CN, Hung YJ, Kwok CF, Sun JH, Pei D, Yang CY, Chen CC, Lin CL and Sheu WH; acarbose/metformin fixed-dose combination study investigators: Acarbose plus metformin fixed-dose combination outperforms acarbose monotherapy for type 2 diabetes. Diabetes Res Clin Pract 102: 16-24, 2013 (In Chinese).

37. Wang Z: Metformin in combination with acarbose in the treatment of type 2 diabetes. Healthy Way 12: 139, 2013 (In Chinese).

38. Wu SY: Acarbose combined with metformin in the treatment of type 2 diabetes. Diabetes New World 21: 5-6, 2014 (In Chinese).
39. Wu XF: Efficacy of metformin with acarbose in the treatment of 105 cases of type 2 diabetes. China Pharmaceuticals 22: 35-36, 2013 (In Chinese).

40. Yang LX: Explore metformin and acarbose clinical curative effect for the treatment of type 2 diabetes. Healthy Way 1: 53 , 2015 (In Chinese).

41. Zhang HL: Metformin in combination with acarbose for treatment of overweight and obese type 2 diabetes. Diabetes New World 20: 24-26, 2014 (In Chinese).

42. Zhang MH: Metformin and acarbose on the treatment of type 2 diabetes insulin resistance. Chinese J Jilin Med 32: 3682-3683, 2011 (In Chinese).

43. Zhang XH and Zhang H: Curative effect observation of different drugs combined therapy with metformin foe incipient type 2 diabetes. Diabetes New World 8: 14, 2014 (In Chinese).

44. Zheng Q and Huangfu YH: Clinical observation of acarbose in combination with metformin for the treatment of type 2 diabetes. J China Pharmacy 24: 2251-2253, 2014 (In Chinese).

45. Zhou XH, Zhong Q and Ye D: The efficacy of acarbose combined with metfIrmin in treatment of type 2 diabete. China Modern Doctor 51: 68-70, 2013 (In Chinese)

46. Zhu ZL, Qiu XC, Zhu KP and Ding ZJ: Application of acarbose combined with metformin in treatment of newly diagnosed type 2 diabetes patients. Heilongjiang Med J 24: 223-225, 2011 (In Chinese)

47. Cheng AY and Josse RG: Intestinal absorption inhibitors for type 2 diabetes mellitus: Prevention and treatment. Drug Discovery Today Therapeutic Strategies 1: 201-206, 2004.

48. Musi N, Hirshman MF, Nygren J, Svanfeldt M, Bavenholm P, Rooyackers O, Zhou G, Williamson JM, Ljunqvist O, Efendic S, et al: Metformin increases AMP-activated protein kinase activity in skeletal muscle of subjects with type 2 diabetes. Diabetes 51: 2074-2081, 2002.

49. Long YC and Zierath JR: AMP-activated protein kinase signaling in metabolic regulation. J Clin Invest 116: 1776-1783, 2006.

50. Hundal RS, Krssak M, Dufour S, Laurent D, Lebon V, Chandramouli V, Inzucchi SE, Schumann WC, Petersen KF, Landau BR and Shulman GI: Mechanism by which metformin reduces glucose production in type 2 diabetes. Diabetes 49: 2063-2069, 2000.

51. Boulé NG, Haddad E, Kenny GP, Wells GA and Sigal RJ: Effects of exercise on glycemic control and body mass in type 2 diabetes mellitus: A meta-analysis of controlled clinical trials. JAMA 286: 1218-1227, 2001.

52. Amori RE, Lau J and Pittas AG: Efficacy and safety of incretin therapy in type 2 diabetes: Systematic review and meta-analysis. JAMA 298: 194-206, 2007. 